\title{
FINITELY BOOLEAN REPRESENTABLE VARIETIES
}

\author{
EMIL W. KISS
}

\begin{abstract}
This paper gives a short, elementary proof of a result of Burris and McKenzie [2] stating that each variety Boolean representable by a finite set of finite algebras is the join of an abelian and a discriminator variety. An example showing that the Boolean product operator $\Gamma^{a}$ is not idempotent is included as well.
\end{abstract}

1. Introduction. The result just mentioned was obtained as a corollary of the authors' description of locally finite decidable varieties with modular congruence lattices, and it was asked in Freese and McKenzie [3] whether there is a "reasonable" proof of this statement. We hope that the one given in the next section is such. In fact, we prove a bit more.

THEOREM. Let $V$ be a variety generated by a finite set $\mathcal{K}$ of finite algebras with the property that the countable members of $V$ are in $\Gamma^{a}(\mathcal{K})$. Then $V$ is the join of an abelian and a discriminator variety (which are independent).

We assume that the reader is familiar with the concept of modular commutator as well as that of Boolean product; these notions together with a complete background of the problem are found in Freese and McKenzie [3].

2. The proof. We fix a variety $V$ satisfying the conditions of the Theorem. By the results of McKenzie [5] (also mentioned in [3]), we may assume that $V$ is congruence permutable and each directly indecomposable algebra of $V$ is finite, and is either simple or abelian. Our reasoning is based on the following concept.

DEFINITION. A subalgebra $\mathfrak{A}$ of an algebra $\mathfrak{B}$ is called very skew if $\mathfrak{A}$ is skew in each direct decomposition of $\mathfrak{B}$, that is, for nontrivial congruences $\theta, \psi$ of $\mathfrak{B}$ with $\theta \circ \psi=1$, $\theta \cdot \psi=0$ we have

$$
(\theta \uparrow \mathfrak{U}) \circ(\psi \uparrow \mathfrak{A})<1_{\mathfrak{A}} .
$$

First, we show that a variety is of the desired type iff the powers of its neutral simple algebras have no large very skew subalgebras.

LEMMA 1. Let $V$ be a finitely generated modular variety with the property that each directly indecomposable member of $V$ is either simple or abelian. Then $V$ is the join of an abelian and a discriminator variety iff for each neutral simple member $\mathfrak{B}_{0}$ of $V$ there exists a natural number $k$ such that the very skew subalgebras of the finite direct powers of $\mathfrak{B}_{0}$ admit at most $k$ elements.

Received by the editors December 30, 1982.

1980 Mathematics Subject Classification. Primary 08B10; Secondary 03B25. 
Before proving this statement, we borrow an observation from [4] (where all modular varieties with complemented principal congruences are described) which will be useful later.

Lemma 2. Let the algebra (5 (in a modular variety) be a subdirect product of some neutral simple algebras ${ }_{\Gamma_{i}}(i \in I)$, and let $\theta, \psi$ be complements in the congruence lattice of 5 . Then for some subsets $A$ and $B=I-A$ of $I$, the congruences $\theta$ and $\psi$ are just the kernels of the projections to $A$ and $B$, respectively.

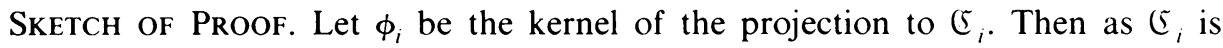
neutral and simple, each $\phi_{i}$ is either over $\theta$ or over $\psi$. Now the choice $A=\left\{i \mid \phi_{i} \geqslant \theta\right\}$ works by modularity.

Let us now prove Lemma 1 . The 'only if' part is clear by standard arguments (see [6]), and by [1, Corollary 9.9] it suffices to show that if $\mathcal{B}_{0}$ is a neutral simple algebra of $V$, and $\mathfrak{H}_{0}$ is a nonsingleton subalgebra of $\mathfrak{B}_{0}$, then $\mathfrak{A}_{0}$ is neutral and simple.

First, let $\phi$ be a nontrivial congruence of $\mathfrak{A}_{0}, \mathfrak{B}=n\left(\mathfrak{B}_{0}\right)$, and set

$$
\mathfrak{H}=\left\{\mathbf{b} \in \in^{n}\left(\mathfrak{U}_{0}\right) \mid b_{i} \phi b_{j}(i, j \in n)\right\} \leqslant \mathscr{H}
$$

(this construction is standard in commutator theory). Now $\mathfrak{A}$ is very skew if $\phi \neq 1$ (since the direct decompositions of $\mathcal{B}$ are the obvious ones by Lemma 2 ), and if $\phi \neq 0$, then $\mathfrak{A}$ has at least $2^{n}$ elements, which is a contradiction.

Secondly, suppose the $\mathfrak{H}_{0}$ is abelian, set $\mathfrak{H}^{2 n}\left(\mathfrak{R}_{0}\right)$ and

$$
\mathfrak{A}=\left\{\mathbf{b} \in^{2 n}\left(\mathfrak{A}_{0}\right) \mid b_{0}+\cdots+b_{n-1}=b_{n}+\cdots+b_{2 n-1}\right\},
$$

where + is an abelian group addition on $\mathfrak{H}_{0}$ compatible with the fundamental operations. This $\mathfrak{A}$ is a subalgebra of $\mathfrak{B}$ of cardinality $\left|\mathfrak{A}_{0}\right|^{2 n-1}$. Furthermore, the images of $\mathscr{H}$ and ${ }^{2 n}\left(\mathfrak{H}_{0}\right)$ are the same in each proper factor of $\mathfrak{B}$, thus $\mathfrak{A}$ is again very skew.

The appropriate sensitive tool for investigating very skew subalgebras seems to be the following.

DEFINITION. Let $\mathfrak{U} \leqslant \mathscr{H}$ be algebras. Set

$$
\mathfrak{B}_{[\mathfrak{H}]}=\left\{u \in{ }^{\omega} \mathfrak{B} \mid \exists a \in \mathfrak{A}:\left\{i \mid u_{i} \neq a\right\} \text { is finite }\right\} .
$$

We denote this element $a$ by $\hat{u}$, and for $b \in \mathcal{B}$ let $\bar{b}: \omega \rightarrow \mathfrak{B}$ be the constant $b$ mapping.

It will turn out that $\mathfrak{A}$ "splits nicely" if we make direct decompositions of $\mathfrak{B}$ with the aid of a Boolean product representation of $\mathfrak{B}_{[\mathfrak{H}]}$. More precisely, we show that if $\mathfrak{B}={ }^{n}\left(\mathfrak{B}_{0}\right)$ for some neutral simple (finite) $\mathfrak{B}_{0}$, and $\mathfrak{U} \leqslant \mathfrak{B}$ is very skew, then $\mathfrak{B}_{[\mathfrak{H}]}$ has only trivial direct decompositions, and hence if $\mathfrak{B}_{[\mathfrak{K}]}$ is in $\Gamma^{a}(\mathcal{K})$, then some element of $\mathscr{K}$ majorates $\mathfrak{A}$ in power. Let us fix the algebras $\mathfrak{B}_{0}, \mathfrak{A}, \mathfrak{B}$ as in the previous sentence.

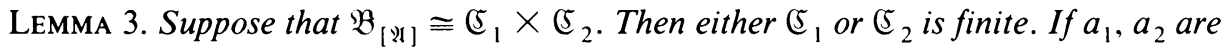
different elements of $\mathfrak{A}$ then the images of $\bar{a}_{1}$ and $\bar{a}_{2}$ in the "cofinite" component of this direct decomposition are also different. 
Proof. We have a subdirect decomposition

$$
\mathfrak{B}_{[\mathfrak{H}]} \leqslant \prod_{n \times \omega} \mathfrak{B}_{0} .
$$

Let $\theta, \psi$ be the congruences corresponding to the decomposition $\mathfrak{B}_{[\mathfrak{M}]} \cong \mathfrak{S}_{1} \times \mathfrak{S}_{2}$, and $A, B \subseteq n \times \omega$ be the subsets given by Lemma 2 . It clearly suffices to show that either $A$ or $B$ is finite.

For each $i \in \omega$ we get a direct decomposition of $\mathfrak{B}$ from that of $\mathfrak{B}_{[\mathfrak{M}]}$ : it is determined by the subsets

$$
A_{i}=\{j \in n \mid(j, i) \in A\} \quad \text { and } \quad B_{i}=\{j \in n \mid(j, i) \in B\}
$$

of $n$. We prove that disregarding finitely many indices $i$, this decomposition is trivial. Indeed, otherwise, there is an infinite $I \subseteq \omega$ such that $A_{i}$ and $B_{i}$ are the same subsets, say $A^{\prime}$ and $B^{\prime}$, of $n$, respectively, for $i \in I$; and $A^{\prime}, B^{\prime} \neq \varnothing$. Let $\theta^{\prime}, \psi^{\prime}$ denote the congruences of $B$ corresponding to its direct decomposition determined by $A^{\prime}$ and $B^{\prime}$. As $\mathfrak{A}$ is very skew, there exist elements $a_{1}, a_{2}$ of $\mathfrak{A}$ such that

$$
\left(a_{1}, a_{2}\right) \notin\left(\theta^{\prime} \uparrow \mathfrak{A}\right) \circ\left(\psi^{\prime} \uparrow \mathfrak{A}\right) \text {. }
$$

On the other hand, $\left(\bar{a}_{1}, \bar{a}_{2}\right) \in \theta \circ \psi$, say $\bar{a}_{1} \theta u \psi \bar{a}_{2}$, and as $I$ is infinite, we clearly have $a_{1} \theta \hat{u} \psi^{\prime} a_{2}$, which is a contradiction.

Suppose now that $A_{i}=\varnothing$, as well as $B_{i}=\varnothing$, hold infinitely many times. Choose arbitrary elements $a_{1} \neq a_{2}$ from $\mathfrak{A}$. Then with some $\bar{a}_{1} \theta u \psi \bar{a}_{2}$ we clearly have $a_{1}=\hat{u}=a_{2}$, which is a contradiction. Thus, either $A$ or $B$ is finite, as desired.

The proof of the Theorem will be complete by showing

LEMma 4. If $\mathfrak{B}_{[\mathfrak{W}]} \in \Gamma^{a}(\mathfrak{K})$, then there exists a $\mathfrak{R} \in \mathfrak{K}$ such that $|\mathfrak{U}| \leqslant|\mathfrak{K}|$.

Proof. We have

$$
\mathfrak{B}_{[\mathfrak{W}]} \leqslant \prod_{h p} \mathfrak{\Re}_{i \in I} .
$$

If for some $i \in I$, the $i$ th components of the elements $\bar{a}(a \in \mathfrak{A})$ are all different, then clearly $|\mathfrak{A}| \leqslant\left|\mathfrak{R}_{i}\right|$. Otherwise, we have (with $\llbracket x=y \rrbracket$ being the equalizer of $x$ and $y$ )

$$
\bigcup_{a_{1} \neq a_{2} \in \mathfrak{A}} \llbracket \bar{a}_{1}=\bar{a}_{2} \rrbracket=I .
$$

Thus we obtain a partition of $I$ into the clopen sets $A_{1}, \ldots, A_{s}$ with the property that each $A_{i}$ is covered by some $\llbracket \bar{a}_{1}=\bar{a}_{2} \rrbracket$. This partition defines a direct decomposition of $\mathfrak{B}_{[\mathfrak{H}]}$ which does not satisfy Lemma 3.

3. $\Gamma^{0}$ is not idempotent. If all the elements of $\mathcal{K}$ are either affine or simple, then we have a straightforward proof to the Theorem by constructing a term in $F_{V}(\omega)$ which is the discriminator in each maximal neutral simple algebra of $V$; and each variety representable by any $\mathscr{K}$ can be represented by such a $\mathscr{K}$ by the Theorem and [6]. However, our previous argument shows that we cannot in general assume that $\mathscr{K}$ is so nice. Indeed, let $\mathfrak{B}_{0}$ be the alternating group on five letters, $\mathfrak{A}_{0}$ a two-element subgroup of $\mathfrak{B}_{0}$ and let $\mathfrak{B}, \mathfrak{U}$ be as in the "abelian" construction of the proof of 
Lemma 1 for some $n$ with the property that $|\mathfrak{A}|=2^{2 n-1}>\left|\mathfrak{B}_{0}\right|$. Then, by Lemma 4 , we have

$$
\mathfrak{P}_{[\mathfrak{H}]} \in \Gamma^{a}(\mathfrak{A}, \mathfrak{B})-\Gamma^{a}\left(\mathfrak{H}_{0}, \mathfrak{B}_{0}\right),
$$

and since clearly $\mathfrak{A}, \mathfrak{B} \in \Gamma^{a}\left(\mathfrak{A}_{0}, \mathfrak{B}_{0}\right)$, this example shows also that the operator $\Gamma^{a}$ is not idempotent.

\section{REFERENCES}

1. S. Burris and R. McKenzie, Decidable varieties with modular congruence lattices. Mem. Amer. Math. Soc. 31 (1981), No. 246, 1-65.

2. __ Boolean representable varieties, Mem. Amer. Math. Soc. 31 (1981), No. 246, 67-106.

3. R. Freese and R. McKenzie, The commutator, an overview, preprint, 1981.

4. E. W. Kiss, Skew and complemented congruences, preprint, 1982.

5. R. McKenzie, Narrowness implies uniformity, preprint, 1980.

6. H. Werner, Discriminator algehras, Studien zur Algebra Bd. 6, Akademie Verlag, Berlin, 1978.

Mathematical Institute of the Hungarian ACademy of Sciences, Reáltanoda U. 13-15. 1053. BUDAPEST, HUNGARY 